\title{
BIFURCATIONS OF SECOND ORDER PROBLEMS WITH JUMPING NONLINEARITIES
}

\author{
Pavel Drábek and Milan Kučera
}

\begin{abstract}
In this paper we deal with nonlinear second order boundary value problems for ordinary differential equations including the case of jumping nonlinearities. The set of generalised eigenvalues in the case of nonconstant coefficients is described. It is proved that these generalised eigenvalues are simultaneously bifurcation points of the problem with coefficients also depending on the solution $u=u(x)$.
\end{abstract}

In this paper, we study the global structure of the set $A_{-1}(r)$ of all pairs $(\mu, \nu) \in \mathbf{R}^{2}$ for which the nonlinear boundary value problem

$$
\begin{gathered}
-\left(a(x, u) u^{\prime}\right)^{\prime}-\mu c(x, u) u^{+}+\nu c(x, u) u^{-}=0 \text { on }[0, \pi] \\
u(0)=u(\pi)=0
\end{gathered}
$$

has a solution $\|u\|_{C^{1}}=r$ (for a given $r>0$ ). First, we shall give a characterisation of this set $A_{-1}$ for the problem

$$
-\left(a(x) u^{\prime}\right)^{\prime}-\mu c(x) u^{+}+\nu c(x) u^{-}=0 \text { on }[0, \pi]
$$

with the boundary conditions (2). (In this case $A_{-1}$ does not depend on $r>0$ ). Note that in the case of constant coefficients, the set $A_{-1}$ of generalised eigenvalues can be descrived explicitly (see Fućik) [4], Dancer [2,3]). Further, by using the characterisation of $A_{-1}$ for (3), (2) with $a(x), c(x)$ replaced by $a(x, w(x)), c(x, w(x))$ and applying a fixed point method we shall obtain information about the character of $A_{-1}(r)$ for any $r>0$. Particularly, $A_{-1}(r)$ tends to $A_{1}(0)$ (the set $A_{-1}$ corresponding to (3), (2) with $a(x)=a(x, 0), c(x)=c(x, 0))$ in a certain sense. Any $(\mu, \nu) \in A_{-1}(0)$ is a bifurcation point of (1), (2) (with respect to the line of trivial solutions). Note that the idea concerning using the fixed point method for finding eigenvalues and eigenfunctions of nonlinear problems is the same as in Boccardo [1], where a different problem is studied.

Received 10 April 1987

Copyright C:learance Centre, Inc. Serial-fee code: 0004-9729/88 \$A2.00+0.00. 


\section{Generalised eigenvalues in the CaSe of nonconstant coefficients}

In this section we shall consider the boundary value problem (3), (2). We suppose that $a \in C^{1}([0, \pi]), c \in C([0, \pi]), c(x)>0, m \leqslant a(x) \leqslant M$ for any $x \in[0, \pi]$, where $0<m<M$. For any $k=1,2, \ldots$, write

$$
\begin{aligned}
Z_{k} & =\left\{u \in C^{1}([0, \pi]) ; u \text { has precisely } k-1 \text { simple zeros in }(0, \pi)\right\}, \\
Z_{k}^{+} & =\left\{u \in Z_{k} ; u^{\prime}(0)>0\right\}, \quad Z_{k}^{-}=\left\{u \in Z_{k} ; u^{\prime}(0)<0\right\} .
\end{aligned}
$$

(By a simple zero, we mean $x$ such that $u(x)=0, u^{\prime}(x) \neq 0$.)

Remark 1. Let us consider the eigenvalue problem

$$
\begin{aligned}
-\left(a(x) u^{\prime}\right)^{\prime}-\lambda c(x) u & =0 \text { on }[0, \pi], \\
u(0)=u(\pi) & =0 .
\end{aligned}
$$

Recall that all the eigenvalues of (4), (2) are simple and form a sequence $\left\{\lambda_{n}\right\}_{n=1}^{\infty}$, $0<\lambda_{1}<\lambda_{2}, \ldots, \lim _{n \rightarrow \infty} \lambda_{n}=\infty$. Further, $v_{n} \in Z_{n}$ for the eigenvalues $v_{n}$ corresponding to $\lambda_{n}$. Hence the eigenfunctions corresponding to the first eigenvalue $\lambda_{1}$ do not change sign on $(0, \pi)$; for $n=2 k$, the eigenfunction $v_{n}$ corresponding to $\lambda_{n}$ consists of $k$ positive and $k$ negative semi-waves; for $n=2 k+1$, there is an eigenfunction $v_{n} \in Z_{k}^{+}$ consisting of $k+1$ positive and $k$ negative semi-waves.

Remark 2. Consider the initial-value problem

$$
\begin{gathered}
-\left(a(x) u^{\prime}\right)^{\prime}-\mu c(x) u^{+}+\nu c(x) u^{-}=0 \text { on }[0, \infty), \\
u(0)=0, \quad u^{\prime}(0)=1 .
\end{gathered}
$$

If $\alpha, \beta$ are two successive zeros of a solution of (5), (6) then on, $[\alpha, \beta],(5)$ reads either as

$$
-\left(a(x) u^{\prime}\right)^{\prime}-\mu c(x) u^{+}=0
$$

or as

$$
-\left(a(x) u^{\prime}\right)^{\prime}+\nu c(x) u^{-}=0
$$

where $u^{+}=u$ or $u^{-}=-u$, respectively. The following assertions give information about how the distance between zeros of the solution of (5), (6) depend on $\mu, \nu$. 
Lemma 1. For $\mu>0, \nu>0$ set

$d^{+}(\mu)=\sup \{|\alpha-\beta| ; \quad \alpha, \beta$ are successive zeros of $u, u$ satisfies $(7)$ on $[\alpha, \beta]\}$,

$d^{-}(\nu)=\sup \{|\alpha-\beta| ; \alpha, \beta$ are successive zeros of $u, u$ satisfies $(8)$ on $[\alpha, \beta]\}$.

Then $d^{+}(\mu) \rightarrow 0$ or $d^{-}(\nu) \rightarrow 0$ if and only if $\mu \rightarrow+\infty$ or $\nu \rightarrow+\infty$, respectively.

Remark 3. Let us write $d_{a, c}^{ \pm}(\mu)$ instead of $d^{ \pm}(\mu)$ in Lemma 1 in order to emphasise the dependence on the coefficients in (7) or (8). Then the convergence $d_{a, c}^{+}(\mu) \rightarrow 0$ for $\mu \rightarrow \infty$ and $d_{a, c}^{-}(\nu) \rightarrow 0$ for $\nu \rightarrow+\infty$ is uniform with respect to $a, c$ satisfying $m \leqslant a(x) \leqslant M, c(x) \geqslant c_{0}$ (with some $0<m<M, c_{0}>0$ fixed).

LEMMA 2. (1) Let us suppose that $u_{i}$ satisfy

$$
-\left(a(x) u_{i}^{\prime}\right)^{\prime}-\mu_{i} c(x) u_{i}^{+}=0
$$

$u_{i}(x)>0$ on $(\alpha, \beta) \subseteq(0, \pi), i=1,2, u_{1}(\alpha)=u_{1}(\beta)=0, u_{2}(\alpha)+u_{2}(\beta)>0$. Then $\mu_{2}<\mu_{1}$.

(2) Let $u_{i}$ satisfy

$$
\begin{gathered}
-\left(a(x) u_{i}^{\prime}\right)^{\prime}+\nu_{i} c(x) u_{i}^{-}=0, \\
u_{i}(x)<0 \text { on }(\alpha, \beta) \subseteq(0, \pi), i=1,2, u_{1}(\alpha)=u_{1}(\beta)=0, u_{2}(\alpha)+ \\
u_{2}(\beta)<0 \text {. Then } \nu_{2}<\nu_{1} .
\end{gathered}
$$

The proofs of Lemmas 1,2 and Remark 3 follow directly from the Sturm comparison Theorem (see Kamke [5]).

LEMMA 3. Let us suppose that

$$
\begin{gathered}
-\left(a(x) u_{i}^{\prime}\right)^{\prime}-\mu_{i} c(x) u_{i}^{+}+\nu_{i} c(x) u_{i}^{-}=0 \text { on }[0, \pi] \\
u_{i}(0)=u_{i}(\pi)=0
\end{gathered}
$$

$i=1,2$. If $\operatorname{sign} u_{1}^{\prime}(0)=\operatorname{sign} u_{2}^{\prime}(0)(\neq 0)$ and $\mu_{1} \leqslant \mu_{2}, \nu_{1} \leqslant \nu_{2}$, with at least one strict inequality sign then $u_{1}$ and $u_{2}$ cannot have the same nonzero number of zeros in $(0, \pi)$.

Proof: Let us suppose that $u_{1}, u_{2} \in Z_{n}^{+}$for some $n=2,3, \ldots$ and $\mu_{1} \leqslant \mu_{2}$, $\nu_{1} \leqslant \nu_{2}$ with at least one strict inequality sign. Elementary considerations give the existence on an interval $(\alpha, \beta) \subset(0, \pi)$ such that $u_{1}(\alpha)=u_{1}(\beta)=0,\left|u_{2}(\alpha)\right|+\left|u_{2}(\beta)\right|>$ 0 and $u_{1}(x) \cdot u_{2}(x)>0$ on $(\alpha, \beta)$. But this contradicts Lemma 2 . 
LEMMA 4. Let $u \in Z_{n}$ be the solution of (3), (2).

(i) If $n=2 k, k=1,2, \ldots$, then $\mu>\lambda_{k}, \nu>\nu_{k}$;

(ii) if $n=2 k+1, k=1,2, \ldots$, then either $\mu>\lambda_{k+1}, \nu>\lambda_{k}$ (in the case $u^{\prime}(0)>0$ ) or $\mu>\lambda_{k}, \nu>\lambda_{k+1}$ (in the case of $\left.u^{\prime}(0)<0\right)$.

ProOF: Let $n=2 k+1, u^{\prime}(0)>0$. Elementary considerations, together with Remark 1, imply that there exist intervals $\left(\alpha_{k}, \beta_{k}\right),\left(\alpha_{k+1}, \beta_{k+1}\right)$ and eigenfunctions $v_{k}, v_{k+1}$ of (4), (2) corresponding to $\lambda_{k}, \lambda_{k+1}$ respectively, such that $u\left(\alpha_{k}\right)=u\left(\beta_{k}\right)=0, v_{k}\left(\alpha_{k}\right)+v_{k}\left(\beta_{k}\right)<0, u(x)<0, v_{k}(x)<0$ for $x \in\left(\alpha_{k}, \beta_{k}\right)$, and $u\left(\alpha_{k+1}\right)=u\left(\beta_{k+1}\right)=0, v_{k+1}\left(\alpha_{k+1}\right)+v_{k+1}\left(\beta_{k+1}\right)>0, u(x)>0, v_{k+1}(x)>0$ for $x \in\left(\alpha_{k+1}, \beta_{k+1}\right)$. The assertion follows from Lemma 2. The other cases can be treated similarly.

Introduce the sets

$$
\begin{aligned}
& A_{-1}=\left\{(\mu, \nu) \in \mathbb{R}^{2} ;(3),(2) \text { has a nontrivial solution }\right\}, \\
& A_{-1}^{+}=\left\{(\mu, \nu) \in \mathbb{R}^{2} ;(3),(2) \text { has a solution in } Z_{n}^{+}\right\}, \\
& A_{-1}^{-}=\left\{(\mu, \nu) \in \mathbb{R}^{2} ;(3),(2) \text { has a solution in } Z_{n}^{-}\right\}, \quad n=1,2, \ldots
\end{aligned}
$$

\section{Theorem 1.}

$$
\begin{aligned}
A_{-1} & =\bigcup_{n=1}^{\infty}\left[A_{n}^{+} \cup A_{n}^{-}\right], \\
A_{1}^{+} & =\left\{(\mu, \nu) \in \mathbb{R}^{2} ; \mu=\lambda_{1}, \nu \text { is arbitrary }\right\} \\
A_{n}^{+} & =\left\{(\mu, \nu) ; \nu=f_{n}(\mu)\right\}, \quad n=2,3, \ldots, \\
A_{n}^{-} & =\left\{(\mu, \nu) ;(\nu, \mu) \in A_{n}^{+}\right\}, \quad n=1,2, \ldots,
\end{aligned}
$$

where $f_{n}$ are continuous decreasing functions such that:

(i) if $n=2 k, k=1,2, \ldots$, then $f_{n}$ is defined on $\left(\lambda_{k}, \infty\right), \lim _{\mu \rightarrow \lambda_{n}^{+}} f_{n}(\mu)=$ $+\infty, \lim _{\mu \rightarrow \infty} f_{n}(\mu)=\lambda_{k}$ and $f_{n}\left(\lambda_{n}\right)=\lambda_{n}$;

(ii) if $n=2 k+1, k=1,2, \ldots$ then $f_{n}$ is defined on $\left(\lambda_{k+1},+\infty\right)$, $\lim _{\mu \rightarrow \lambda_{k+1}^{+}} f_{n}(\mu)=+\infty, \lim _{\mu \rightarrow \infty} f_{n}(\mu)=\lambda_{k}$ and $f_{n}\left(\lambda_{n}\right)=\lambda_{n}$.

ProOf: The first equality follows from the fact that any nontrivial solution of (3), (2) has a finite number of simple zeros, by the uniqueness of solutions to the initial value problem for ODE's. The second equality is clear because $u$ is a positive or negative solution of (3), (2) if and only if it is the eigenfunction of (4), (2) corresponding to the first eigenvalue. Further let $n=2 k+1$ for some $k=1,2, \ldots$. Let $\mu \in\left(\lambda_{k+1}, \infty\right)$ be arbitrary but fixed. Denote by $u_{\mu, \nu}$ the solution of the initial value problem $(5),(6)$ 
and by $x_{n}^{\mu, \nu}$ the $n$-th zero of $u_{\mu, \nu}$ in $(0, \infty)$. Lemmas 1,2 , together with Remark 1 , imply that $x_{n}^{\mu, \nu}<\pi$ if $\nu$ is large enough. On the other hand, $x_{n}^{\mu, \nu}>\pi$ for $\nu$ small by the Strum comparison Theorem (see [5]). Hence, there exists at least one $\nu>0$ such that $x_{n}^{\mu, \nu}=\pi$ according to the continuous dependence of $u_{\mu, \nu}$ on the parameters $\mu$, $\nu$. For this $\nu, u_{\mu, \nu}$ is the solution of the boundary value problem (3), (2), $u_{\mu, \nu} \in Z_{n}^{+}$. Lemma 4 (ii) implies that $\nu \in\left(\lambda_{k}, \infty\right)$ and Lemma 3 ensures the uniqueness of $\nu$. Hence, for any $\mu \in\left(\lambda_{k+1}, \infty\right)$ we can set $f_{n}(\mu)=\nu$ where $\nu$ is such that (3), (2) has a solution $u_{\mu, \nu} \in Z_{n}^{+}$. In the same way as above, we can prove that for any $\nu \in\left(\lambda_{k}, \infty\right)$ there exists exactly one $\mu \in\left(\lambda_{k+1}, \infty\right)$ such that (3), (2) has a nontrivial solution $u_{\mu, \nu} \in Z_{n}^{+}$. Hence, $f_{n}$ maps $\left(\lambda_{k+1}, \infty\right)$ onto $\left(\lambda_{k}, \infty\right)$ and it is decreasing by Lemma 3. It follows that $f_{n}$ is also continuous and $\lim _{\mu \rightarrow \lambda_{k+1}^{+}} f_{n}(\mu)=+\infty, \lim _{\mu \rightarrow+\infty} f_{n}(\mu)=\lambda_{k}$.

Since (3), (2) has no solution $u \in Z_{n}^{+}$if $\mu \leqslant \lambda_{k+1}$ according to Lemma 2, the assertion concerning $A_{n}^{+}$, with $n=2 k+1$ is proved. Analogously for $A_{n}^{-}$. The proof for $n=2 k$, $k=1,2, \ldots$, can be performed in a similar way.

\section{GENERAL CASE, BIFURCATIONS}

Let us suppose that $a(x, s) \in C^{1}([0, \pi] \times \mathbf{R}), c(x, s) \in C([0, \pi] \times \mathbf{R}), m \leqslant a(x, s) \leqslant$ $M, c(x, s)>0$ for all $x, s \in[0, \pi] \times \mathbf{P}$ with some $M>m>0$. Set $C^{1}=C^{1}([0, \pi])$, $C_{0}^{2}=\left\{u \in C^{2}([0, \pi]) ; u(0)=u(\pi)=0\right\}$ and denote by $\|\cdot\|_{i}$ the usual norm in $C^{i}([0, \pi])$. If $w \in C^{1}$ then $a_{w}(x):=a(x, w(x)), c_{w}(x):=c(x, w(x))$ satisfy all the assumptions imposed on $a$ and $c$ at the begining of Section 1. Further $c_{w}(x) \geqslant \gamma(r)$ for any $x \in[0, \pi]$ and $w \in C^{1},\|w\|_{1} \leqslant r$ with some $\gamma(r)>0$ depending only on $r$.

Remark 4. Let us denote by $\lambda_{n}(w)$ the $n$-th eigenvalue of (4), (2) where $a$ and $c$ are replaced by $a_{w}$ and $c_{w}$. Set

$$
\lambda_{n}^{M}(r)=\inf \left\{\lambda_{n}(w) ;\|w\|_{1} \leqslant r\right\}, \quad \lambda_{n}^{m}(r)=\sup \left\{\lambda_{n}(w) ;\|w\|_{1} \leqslant r\right\},
$$

It follows from the continuous dependence of the eigenvalues of (4), (2) on the coefficients that $\lim _{r \rightarrow 0+} \lambda_{n}^{M}(r)=\lim _{r \rightarrow 0+} \lambda_{n}^{m}(r)=\lambda_{n}(0)$. The variational characterisation of $\lambda_{n}(w)$ ensures that $\lambda_{n}^{M} \leqslant \lambda_{n}^{M}(r), \lambda_{n}^{m} \leqslant \lambda_{n}^{m}(r)$ for all $r>0$ with some $\lambda_{n}^{m}>\lambda_{n}^{M}>0$.

Remark 5. Let $A_{n}^{ \pm}(w), n=1,2, \ldots$, be sets from Theorem 1 for (3), (2) with $a_{w}$, $c_{w}$ instead of $a, c$. If $n=2 k, w \in C^{1},\|w\|_{1} \leqslant r$ and $\mu \in\left(\lambda_{k}^{m}(r),+\infty\right)$ Then there is precisely one $\nu_{+}$and $\nu_{-}$such that $\left(\mu, \nu_{+}\right) \in A_{n}^{+}(w)$ and $\left(\mu, \nu_{-}\right) \in A_{n}^{-}(w)$. If $n=2 k+1, w \in C^{1},\|w\|_{1} \leqslant r$ and $\mu \in\left(\lambda_{k}^{m}(r),+\infty\right)$ or $\mu \in\left(\lambda_{k+1}^{m}(r),+\infty\right)$ then there is precisely one $\nu_{+}$or $\nu_{-}$such that $\left(\mu, \nu_{+}\right) \in A_{n}^{+}(w)$ or $\left(\mu, \nu_{-}\right) \in A_{n}^{-}(w)$, respectively (see Theorem 1). In any case, the set

$$
\left\{\nu \in \mathbf{R} ;(\mu, \nu) \in A_{n}^{+}(w) \text { for some } w \in C^{1},\|w\|_{1} \leqslant r\right\}
$$


is bounded for any $r>0$ and $\mu$ fixed from the corresponding interval just described. Analogously for $A_{n}^{-}(w)$. The last assertion follows from Lemma 1 and Remark 3 .

LEMMA 5. Let $z_{n} \in C_{0}^{2}$ satisfy

$$
-\left(a\left(x, w_{n}\right) z_{n}^{\prime}\right)^{\prime}=f_{n}
$$

and $z \in C_{0}^{2}$ be the solution of

$$
-\left(a(x, w) z^{\prime}\right)^{\prime}=f
$$

Suppose that $w_{n} \rightarrow w$ in $C_{1}$ and $f_{n} \rightarrow f$ in $C([0, \pi])$. Then $z_{n} \rightarrow z$ in $C_{0}^{2}$.

Proof: Put

$$
L_{n}(v)=-\left(a\left(x, w_{n}\right) v^{\prime}\right)^{\prime}, \quad L(v)=-\left(a,(x, w) v^{\prime}\right)^{\prime}
$$

Since $w_{n} \rightarrow w$ in $C^{1}([0, \pi])$ we have $L_{n}(v) \rightarrow L(v)$ in $C([0, \pi])$, for any $v \in C_{0}^{2}$. Hence

$$
\begin{aligned}
\left\|z_{n}-z\right\|_{2} & =\left\|L_{n}^{-1}\left(f_{n}\right)-L^{1}(f)\right\|_{2} \\
& \leqslant\left\|L_{n}^{-1}\left(f_{n}\right)-L_{n}^{-1}(f)\right\|_{2}+\left\|L_{n}^{-1}\left(L_{n}-L\right) L^{-1}(f)\right\|_{2} \\
& \leqslant c\left(\left\|f_{n}-f\right\|_{0}+\left\|\left(L_{n}-L\right)\left(L^{-1}(f)\right)\right\|_{0}\right),
\end{aligned}
$$

with $c>0$ independently of $n$. (The boundedness of $L_{n}^{1}$ follows by elementary considerations.)

Theorem 2. Let $r>0$ be arbitrary but fixed

(i) If $n=2 k, k=1,2, \ldots$, then for any $\mu \in\left(\lambda_{k}^{m}(r), \infty\right)$ we can find $\nu \in\left(\lambda_{k}^{M}(r), \infty\right)$ such that (1), (2) has a solution $u \in Z_{n}^{+},\|u\|_{1}=r$. The same holds for $Z_{n}^{-}$.

(ii) If $n=2 k+1, k=1,2, \ldots$ then for any $\mu \in\left(\lambda_{k+1}^{m}(r), \infty\right)$ we can find $\nu\left(\lambda_{k}^{M}(r), \infty\right)$ such that (1), (2) has a solution $u \in Z_{n}^{+},\|u\|_{1}=r$ and for any $\mu \in\left(\lambda_{n}^{m}(r), \infty\right)$ we can find $\nu \in\left(\lambda_{k+1}^{M}(r), \infty\right)$ such that (1), (2) has a solution $u \in Z_{n}^{-},\|u\|_{1}=r$.

(iii) If $n=1$ then there exists $\mu_{r} \in\left[\lambda_{1}^{M}(r), \lambda_{n}^{m}(r)\right]$ such that there is $u \in Z_{1}^{+}$ satisfying (1), (2) with $\mu=\mu_{r}$ and any $\nu,\|u\|_{1}=r$, and there exists $\nu_{r} \in\left[\lambda_{1}^{M}(r), \lambda_{1}^{m}(r)\right]$ such that there is $u \in Z_{1}^{-}$satisfying (1), (2) with any $\mu$ and $\nu=\nu_{r},\|u\|_{1}=r$.

Proof: Suppose that $n=2 k$ for some $k=1,2, \ldots$, and that $\mu \in\left(\lambda_{k}^{m}(r), \infty\right)$. For some $w \in C^{1},\|w\|_{1} \leqslant r$, there is a unique $\nu_{w} \in\left(\lambda_{n}(r), \infty\right)$ such that $\left(\mu, \nu_{w}\right) \in$ 
$A_{n}^{+}(w)$ (see Theorem 1). Denote by $B_{r}$ the closed ball in $C^{1}$ with the radius $r$ centred at the origin. Define the mapping $S: B_{r} \rightarrow B_{r}$ which associates with any fixed $w \in B_{r}$ the function $v$ satisfying

$$
\begin{gathered}
-\left(a(x, w) v^{\prime}\right)^{\prime}-\mu c(x, w) v^{+}+\nu_{w} c(x, w) v^{-}=0 \text { on }[0, \pi] \\
v(0)=v(\pi)=0, \quad v \in Z_{n}^{+}, \quad\|v\|_{1}=r .
\end{gathered}
$$

Theorem 1, positive homogeneity of (14) (with respect to $v$ ) and the uniqueness theorem for second order ODE's imply that $S$ is well-defined. Let us prove that $S$ is a continuous mapping from $C^{1}$ into $C_{0}^{2}$. Suppose that $w_{m} \rightarrow w$ in $C^{1}$ and set $v_{m}=S\left(w_{m}\right)$, $v=S(w), \nu_{m}=\nu_{w_{m}}$. The Arselá-Ascoli theorem and Remark 5 imply that there are subsequences (denoted again by $\left.\left\{v_{n}\right\},\left\{\nu_{n}\right\}\right)$ such that $v_{n} \rightarrow \bar{v}$ in $C([0, \pi]), \nu_{n} \rightarrow \bar{\nu} \in$ $\left[\lambda_{k}^{M}(r), \infty\right)$. Hence

$$
\mu c\left(x, w_{n}\right) v_{n}^{+}-\nu_{n} c\left(x, w_{n}\right) v_{n}^{-} \rightarrow \mu c(x, w) \bar{v}^{+}-\bar{\nu} c(x, w) \bar{v}^{-} \text {in } C([0, \pi]) .
$$

It follows from Lemma 5 that $v_{n} \rightarrow \bar{v}$ in $C^{2}$, that is, $\bar{v}$ satisfies (14). Further, $\bar{v} \in Z_{n}^{+}$because $v_{n} \in Z_{n}^{+}$. (In the opposite case $\bar{v}$ would have a double zero which is impossible with respect to the uniqueness of solutions to the initial value problem for ODE's.) Theorem 1 ensures that there are unique $\nu_{w}$ and $v$ satisfying (14), (15). This implies $\nu=\bar{\nu}, v_{n} \rightarrow \bar{v}=v$ for the whole sequence $\left\{v_{n}\right\}$ and the continuity of $S: B_{r} \rightarrow C_{0}^{2}$ is proved. Now, we can apply the Schauder fixed point theorem in $B_{r}$. Hence, for any $\mu \in\left(\lambda_{k}^{m}(r), \infty\right)$ we get $\nu \in\left(\lambda_{k}^{M}(r), \infty\right), u \in Z_{n}^{+}$satisfying (1), (2) and $\|u\|_{1}=r$.

The proof of (ii) can be performed in the same way. If $n=1$ then for $r>0$ fixed we define the mapping $S: B_{r} \rightarrow B_{r}$ which associates with any fixed $w \in H$ the solution of

$$
\begin{gathered}
-\left(a(x, w) v^{\prime}\right)^{\prime}-\lambda_{1}(w) c(x, w) v=0 \text { on }[0, \pi] \\
v(0)=v(\pi)=0, \quad v>0 \text { in }(0, \pi), \quad\|v\|_{1}=r
\end{gathered}
$$

In the same way as above we prove the continuity of $S$ from $C^{1}$ into $C_{0}^{2}$. The fixed point $u$ of $S$ is the positive solution of (1), (2) with $\mu=\lambda_{1}(u)$ satisfying $\|u\|_{1}=r$. If we replace $\mu$ by $\nu$ and $v>0$ by $v<0$ we get the second part of assertion (iii).

Theorem 3. Let $\left(\mu_{0}, \nu_{0}\right) \in A_{n}^{+}(0)$ be fixed. Then for any $r>0$ sufficiently small, there exist $u_{r}, \nu_{r}$ such that $u_{r}$ is the solution of (1), (2) with $\mu=\mu_{0}, \nu=\nu_{r}$, $u_{r} \in Z_{n}^{+},\left\|u_{r}\right\|_{1}=r$. Moreover, $\nu_{r} \rightarrow \nu_{0}, \frac{u_{r}}{\left\|u_{r}\right\|_{1}} \rightarrow u$ (if $r \rightarrow 0+$ ) where $u \in Z_{n}^{+}$is a 
solution to (3), (2) with $a(x)=a(x, 0), c(x)=c(x, 0), \mu=\mu_{0}, \nu=\nu_{0}$, and $\|u\|_{1}=1$. The same assertion holds for $A_{n}^{-}(0)$ (with $Z_{n}^{+}$replaced by $Z_{n}^{-}$).

Proof: If $r>0$ is sufficiently small, then $\mu_{0}>\lambda_{n / 2}^{m}(r)$ (see Theorem 1 and Remark 4). Theorem 2 ensures the existance of $u_{r}, \nu_{r}$ with the presented properties. Put $v_{r}=\frac{u_{r}}{\left\|u_{r}\right\|_{1}}$. Since $\left\|v_{r}\right\|_{1}=1$ there is some sequence $\left\{r_{k}\right\}, r_{k} \rightarrow 0+$ such that $v_{r_{k}} \rightarrow v$ in $C([0, \pi])$ for some $v$ and we can suppose $\nu_{r_{k}} \rightarrow \nu$ for some $\nu \in \mathrm{R}$ by Remark 5. We pass to the limit as $k \rightarrow \infty$ in

$$
-\left(a\left(x, u_{r_{k}}\right) v_{r_{k}}^{\prime}\right)^{\prime}-\mu_{0} c\left(x, u_{r_{k}}\right) v_{r_{k}}^{+}+\nu_{r_{k}} c\left(x, u_{r_{k}}\right) v_{r_{k}}^{-}=0
$$

The last equation and Lemma 5 imply that $v_{r_{k}} \rightarrow v$ in $C_{0}^{2}$ and we obtain

$$
\begin{gathered}
-\left(a(x, 0) v^{\prime}\right)^{\prime}-\mu_{0} c(x, 0) v^{+}+\nu c(x, 0) v^{-}=0 \text { on }[0, \pi] \\
v(0)=v(\pi)=0, \quad v \in Z_{n}^{+}, \quad\|v\|_{1}=1 .
\end{gathered}
$$

Since $v$ and $\nu$ satisfying (18), (19) (with $\mu_{0}$ given) are determined uniquely, we obtain $v_{r} \rightarrow v$ in $C_{0}^{2}, \nu_{r} \rightarrow \nu=\nu_{0}$ (see Theorem 1$)$.

Remark 6. Theorem 3 (together with Theorem 1) ensures that any $\left(\mu_{0}, \nu_{0}\right) \in A_{-1}(0)$ is a bifurcation point of (1), (2). Note that the following more detailed assertion can be proved analoguously to Theorem 3 :

Theorem 4. Let $\left(\mu_{0}, \nu_{0}\right) \in A_{n}^{+}(0)$, let $\Gamma$ be a curve in $\mathrm{R}^{2}$ containing $\left(\mu_{0}, \nu_{0}\right)$ and transversal to $A_{n}^{+}(0)$. Then for any $r>0$ small enough there exist $\left(\mu_{r}, \nu_{r}\right) \in \Gamma$ and a solution $u_{r} \in Z_{n}^{+}$of (1), (2) with $\mu=\mu_{r}, \nu=\nu_{r},\left\|u_{r}\right\|_{1}=r$. Moreover, $\left(\mu_{r}, \nu_{r}\right) \rightarrow\left(\mu_{0}, \nu_{0}\right), \frac{u_{r}}{\left\|u_{r}\right\|_{1}} \rightarrow u$ for $r \rightarrow 0+$ where $u \in Z_{n}^{+}$is a solution to (3), (2) with $a(x)=a(x, 0), c(x)=c(x, 0), \mu=\mu_{0}, \nu=\nu_{0}$ and $\|u\|_{1}=1$. Analogously for $A_{n}^{-}(0)$.

Remark 7. Our result contained in Theorem 2 (iii) concerning strictly positive solutions of (1), (2) is a special case of the result of Boccardo [1].

\section{REFERENCES}

[1] L. Boccardo, 'Positive eigenfunctions for a class of quasilinear operators', Boll. Un. Mat. Ital. 5 (1981), 951-959.

[2] E.N. Dancer, 'On a nonlinear elliptic boundary-value problem', Bull. Austral. Math. Soc. 12 (1975), 399-405.

[3] E.N. Dancer, 'Boundary value problems for weakly nonlinear ordinary differential equations', Bull. Austral. Math. Soc. 15 (1976), 321-328. 
[4] S. Fučik, Solvability of Nonlinear Equations and Boundary Value Problems (D. Reidel Publ Comp., Dordrecht, 1980).

[5] E. Kanke, Differential Gleichungen, Lösungs-methoden und Lösungen, Russian (Nauka, Moscow, 1971).

KMA VŚSE,

Nejedleho Sady 14,

30614 Plzen,

CZECHOSLOVAKIA
MU CSAV

Zitna 25

11567 Praha

CZECHOSLOVAKIA 